\title{
Autopercepção da saúde e desempenho cognitivo em idosos residentes na comunidade
}

\author{
Self-perception of health and cognitive performance in community-dwelling elderly \\ Daniela Helena Machado de Freitas ${ }^{1}$, Fernanda Carolina Alves Campos ${ }^{1}$, Lorena Quintão Linhares ${ }^{1}$, \\ Carolina Ribeiro dos Santos ${ }^{1}$, Cristina Barcelos Ferreira ${ }^{1}$, Breno Satler Diniz ${ }^{2}$, Almir Tavares ${ }^{3}$
}

'Acadêmica do curso de Medicina da Universidade Federal de Minas Gerais (UFMG). Membro do Programa Lar dos Idosos da UFMG, Belo Horizonte, MG.
2Psiquiatra, pesquisador do Laboratório de Neurociências (LIM 27) do Instituto e Departamento de Psiquiatria da Faculdade de Medicina da Universidade de São Paulo (FMUSP), São Paulo, SP.
3 Professor-associado do Departamento de Saúde Mental da Faculdade de Medicina da UFMG. Coordenador do Programa Lar dos Idosos da UFMG, Belo Horizonte, MG.

Recebido: 27/1/2009 - Aceito: 29/4/2009

Resumo
Contexto: A autopercepção de saúde contempla vários aspectos da saúde física, cognição e capacidade funcional. Objetivo: Comparar idosos com e sem evidência
de declínio cognitivo/demência quanto a autopercepção de saúde e variáveis sociodemográficas, destacando as variáveis associadas à autopercepção de saúde
positiva e negativa dentro destes grupos. Métodos: Noventa e oito idosos residentes na comunidade foram avaliados em visitas domiciliares para este trabalho.
A avaliação cognitiva e funcional foi feita por meio da aplicação do Mini-Exame do Estado Mental (MEEM), do Teste do Desenho do Relógio (TDR), da Escala
de Demência de Blessed (EDB) e do Questionário do Informante de Declínio Cognitivo em Idosos (IQCODE). A autopercepção da saúde foi avaliada por in-
termédio de um questionário adaptado do Brazil Old Age Schedule. Resultados: Vinte e seis idosos (26\%) apresentaram quadro de declínio cognitivo. Autoper-
cepção de saúde negativa e menor participação em atividades físicas e recreativas se associaram significativamente a declínio cognitivo nesta amostra (p = 0,006 ,
p = $0,05, p=0,03$, respectivamente). No grupo de sujeitos sem evidência de declínio cognitivo, a história prévia de eventos cerebrovasculares se relacionou à maior
frequência de autopercepção negativa da saúde. Conclusão: A autopercepção negativa da saúde está relacionada ao provável diagnóstico de demência. Nos idosos
com declínio cognitivo, a autopercepção positiva de saúde associa-se a quadros mais graves, refletindo possivelmente menor insight quanto ao estado mórbido.

Freitas DHM, et al. / Rev Psiq Clín. 2010;37(1):32-5

Palavras-chave: Autopercepção de saúde, idoso, envelhecimento, cognição, demência.

\begin{abstract}
Background: The self-perception of health can approach several aspects of physical health, cognition and functional abilities. Objective: To compare elderly subjects wit and without evidence of cognitive decline in relation to health self-perception and socio-demographic variables, highlighting the variables associated with positive or negative health self-perception in these groups. Methods: Ninety eight community-dwelling subjects were assessed in this study. Cognitive and functional abilities were tested using the Mini-Mental State Examination (MMSE), the Clock Drawing Test (CDT), the Blessed Dementia Scale (BDS), and the Informant Questionnaire of Cognitive Decline in the Elderly (IQCODE). Self-perception of health evaluation was performed with the use of an adapted questionnaire of the Brazil Old Age Schedule. Results: Twenty-six subjects (26\%) had evidence of cognitive decline in this sample. Negative health self-perception and lower levels of physical and leisure activity were significantly associated with cognitive decline in this sample ( $p=0.006, p=0.05, p=0.03$, respectively). In the group of subjects without evidence of dementia, history of cerebrovascular disease was associated with negative health perception. Discussion: Dementia was associated with worse health self-perception evaluation. In addition, elders with evidence of dementia and good health self-perception evaluation had higher IQCODE scores, suggesting lack of judgment regarding health status.
\end{abstract}

Freitas DHM, et al. / Rev Psiq Clín. 2010;37(1):32-5

Key-words: Health self-perception, elderly, aging, cognition, dementia.

\section{Introdução}

A avaliação da autopercepção de saúde tem se mostrado método confiável e com validade equivalente a outras medidas mais complexas para a mensuração do estado de saúde de um sujeito, além de ser de fácil aplicação ${ }^{1-2}$. Este método vem sendo frequentemente utilizado na abordagem da associação entre saúde, determinantes demográficos e socioeconômicos, doenças crônicas e capacidade funcional em idosos ${ }^{3}$. A autopercepção de saúde contempla diversos aspectos da saúde física, da cognição e da capacidade funcional de um sujeito ${ }^{4}$. A autopercepção negativa de saúde é um preditor de maior risco de mortalidade, isto é, aqueles com a autopercepção de saúde negativa apresentam maior risco de mortalidade em comparação com uma autopercepção positiva da saúde ${ }^{5-7}$. Segundo Appels et $a l .{ }^{8}$, a autopercepção de saúde se associa fortemente com o estado real ou objetivo de saúde das pessoas e pode ser considerada uma representação das avaliações objetivas de saúde.

Em idosos, a autopercepção de saúde recebe a influência de múltiplas áreas: aspectos demográficos, socioeconômicos, estado de saúde física e capacidade funcional ${ }^{7}$. Em um estudo realizado em São Paulo, SP, a presença de doenças crônicas associadas ao gênero é o determinante mais fortemente relacionado com a autopercepção de saúde dos idosos, vindo, a seguir, a capacidade funcional ${ }^{9}$.

O envelhecimento populacional em países em desenvolvimento é um novo desafio para a saúde pública. De acordo com dados do Instituto Brasileiro de Geografia e Estatística (IBGE) de 2002, de 1991 a 2000, o número de idosos aumentou duas vezes e meia (35\%) em relação ao resto da população $(14 \%)^{10}$. $\mathrm{O}$ aumento da expectativa de vida se associa à maior incidência

Instituição onde o trabalho foi realizado: Programa Lar dos Idosos do Departamento de Saúde Mental da Faculdade de Medicina da Universidade Federal de Minas Gerais. Endereço para correspondência: Breno Satler Diniz. Laboratório de Neurociências - LIM 27. Instituto de Psiquiatria, Hospital das Clínicas, FMUSP. Rua Dr. Ovídio Pires de Campos, 785, $3^{\circ}$ andar 05403-010 - São Paulo, SP. E-mail: brenosatler@usp.br 
de doenças relacionadas ao envelhecimento, como as doenças crônico-degenerativas, cardiovasculares, câncer, além de doenças neurodegenerativas, com destaque para doença de Alzheimer ${ }^{11}$. Sua prevalência média em indivíduos acima de 65 anos varia de acordo com as populações e os critérios diagnósticos, indo desde 2,2\% na África, 5,7\% na Ásia, 6,2\% na América do Norte, 7,1\% na América do Sul até 9,0\% na Europa ${ }^{12}$. No Brasil, a incidência de demência em idosos residentes na comunidade alcança a taxa de 13,8/1.000 habitantes/ano ${ }^{13}$. Os quadros demenciais apresentam alta morbidade e contribuem para a piora do prognóstico de várias outras doenças, além de causar grande ônus biopsicossocial aos familiares e cuidadores ${ }^{14}$.

Há poucos estudos sobre a percepção da saúde na população brasileira ${ }^{15}$. Além disso, não há estudos em nosso meio que avaliaram diretamente a autopercepção da saúde em relação ao desempenho cognitivo em sujeitos com e sem evidência de transtornos cognitivos residentes na comunidade. Portanto, o objetivo deste trabalho é comparar idosos com e sem evidência de declínio cognitivo/demência com relação a autopercepção de saúde e variáveis sociodemográficas, destacando as variáveis associadas à autopercepção de saúde positiva ou negativa dentro desses grupos.

\section{Métodos}

\section{Desenho e casuística}

Este é um estudo transversal e se baseou na coleta de dados obtidos durante visitas domiciliares em uma amostra de conveniência da região oeste de Belo Horizonte, $\mathrm{MG}^{16}$. Todos os sujeitos avaliados tinham acima de 65 anos de idade e residiam em sua residência na época da avaliação. Este trabalho foi aprovado pelo Comitê de Ética em Pesquisa da Universidade Federal de Minas Gerais (UFMG) (ETIC 423/05) e pelo Comitê de Ética em Pesquisa - Secretaria Municipal de Saúde de Belo Horizonte (protocolo 031/2006).

Noventa e oito idosos foram avaliados neste estudo (idade média de 76,9 $\pm 6,0$ anos; escolaridade média de 3,4 $\pm 2,4$ anos). Durante as visitas domiciliares, acadêmicos do curso de medicina da Faculdade de Medicina da UFMG realizaram avaliação clínica breve e aplicaram um questionário baseado no Brazil Old Age Schedule (BOAS $)^{17}$. A avaliação clínica consistia em colher dados da história clínica pregressa (acidente vascular cerebral, traumatismo cranioencefálico, infarto agudo do miocárdio), levantamento dos medicamentos em uso e aferição da pressão arterial.

O BOAS é um instrumento multidimensional que foi validado no Brasil, por meio de pesquisa realizada por Veras ${ }^{18}$, com população idosa, no Rio de Janeiro. Esse instrumento é utilizado em estudos comunitários para traçar o perfil socioeconômico e epidemiológico da população idosa. O questionário é dividido em nove seções: I Informações Gerais, II Saúde Física, III Utilização dos Serviços Médicos e Dentários, IV Atividades da Vida Diária, V Recursos Sociais, VI Recursos Econômicos, VII Saúde Mental, VIII Necessidades e Problemas que Afetam o Entrevistado e IX Avaliação do Entrevistador. Essas seções objetivam assegurar informações sobre as principais características, necessidades e problemas da população idosa de um grande centro urbano ${ }^{19}$. A escala de autopercepção da saúde desta entrevista permite a avaliação do paciente em uma escala qualitativa que varia de muito ruim - ruim - normal - boa - muito boa. Para as análises estatísticas, as respostas foram divididas em autopercepção de saúde positiva (respostas normal, boa e muito boa) ou negativa (respostas ruim e muito ruim).

A avaliação cognitiva e funcional dos idosos foi feita pela aplicação do Short Informant Questionnaire on Cognitive Decline in the Elderly (IQCODE) ${ }^{20,21}$, do Teste do Desenho do Relógio (TDR ${ }^{22}$, da Escala de Demência de Blessed (EDB) ${ }^{23}$ e do Mini-Exame do Estado Mental (MEEM) ${ }^{24}$. O TDR foi pontuado de acordo com as regras propostas por Shulman ${ }^{22}$. Os pontos de corte do MEEM usados para indicar quadro sugestivo de declínio cognitivo foram $<20$ pontos (analfabetos) e $<24$ pontos (idosos escolarizados) ${ }^{25}$. Todas as acadêmicas foram treinadas para a aplicação das entrevistas e escalas desse estudo. De acordo com o padrão de queixas, o histórico médico e o desempenho na avaliação cognitiva, os idosos foram classificados em dois grupos: provável declínio cognitivo/demência (de acordo com os critérios do DSM-IV) e sem declínio cognitivo.

\section{Análises estatísticas}

Dois grupos de análises estatísticas foram realizados para esse trabalho. No primeiro grupo de análises, comparou-se o grupo de sujeitos com e sem evidência de declínio cognitivo com relação a variáveis sociodemográficas (idade, escolaridade, local de nascimento - zona urbana ou rural, renda familiar), dados da história clínica (número de medicamentos em uso, história de doença cerebrovascular, atividade recreativa, atividade física), percepção da saúde (positiva ou negativa) e escores nos testes cognitivos (MEEM, IQCODE, TDR e EDB). No segundo grupo de análises, a variável independente foi a percepção de saúde nessa população. Para isso, os dados sobre autopercepção de saúde foram dicotomizados em percepção positiva/neutra ou negativa. Neste grupo de análises, elas foram realizadas para toda a amostra e também para os grupos diagnósticos individualmente.

Em ambos os grupos de análises, o teste de qui-quadrado foi feito para avaliar a diferença nas distribuições de variáveis dicotômicas entre os grupos de pacientes com e sem demência. O teste $t$ de Student foi realizado para avaliar a diferença entre as médias das variáveis contínuas entre os grupos de pacientes com e sem demência. O cálculo do odds ratio foi realizado por meio da metodologia de Mantel-Haenszel. As análises estatísticas foram feitas com o programa SPSS v.13.0 para Windows (Chicago, IL) e o nível de significância estatística foi definido em $\alpha=5 \%$.

\section{Resultados}

Vinte e seis idosos (26\%) apresentavam diagnóstico de provável declínio cognitivo/demência. Esses eram mais velhos, tinham menor renda familiar e pior desempenho nos testes cognitivos que os sem evidência de declínio cognitivo (Tabela 1). O diagnóstico de provável declínio cognitivo/demência se associou significativamente à autopercepção negativa da saúde $\left(\mathrm{OR}=1,75 \mathrm{CI}_{950}[1.22-2.51]\right.$, $\mathrm{p}=0,008)$, menor participação em atividades físicas ( $\mathrm{OR}=1,38 \mathrm{CI}_{95 \%}$ $[1.03-1.86], \mathrm{p}=0,044)$ e em atividades recreativas $\left(\mathrm{OR}=1,64 \mathrm{CI}_{95 \%}^{95 \%}\right.$ $[1,07-2,51], \mathrm{p}=0,037)$. As demais variáveis não tiveram diferença significativa entre os grupos.

Tabela 1. Variáveis sociodemográficas e escores nos testes cognitivos nos pacientes com demência e sem demência

\begin{tabular}{l|c|c|c|c|c|c|c|c|c}
\hline & Sexo (H/M) & Idade $^{1}$ & Escolaridade & Renda familiar & $\begin{array}{c}\text { Número de } \\
\text { medicações }\end{array}$ & MEEM & TDR & IQCODE & EDB \\
\hline $\begin{array}{l}\text { Declínio } \\
\text { cognitivo } \\
(\mathrm{n}=26)\end{array}$ & $7 / 19$ & $81,2 \pm 7,3^{*}$ & $3,7 \pm 2,6$ & $2,0 \pm 1,0^{*}$ & $3,8 \pm 2,1$ & $16,3 \pm 5,3^{*}$ & $0,8 \pm 0,7^{*}$ & $3,8 \pm 0,7^{*}$ & $4,3 \pm 4,0^{*}$ \\
\hline $\begin{array}{l}\text { Sem declínio } \\
\text { cognitivo } \\
(\mathrm{n}=72)\end{array}$ & $48 / 24$ & $75,3 \pm 4,6$ & $3,3 \pm 2,4$ & $2,8 \pm 1,8$ & $4,0 \pm 2,8$ & $22,8 \pm 3,6$ & $1,9 \pm 1,5$ & $3,2 \pm 0,2$ & $0,7 \pm 0,9$ \\
\hline
\end{tabular}

${ }^{*} p=0,001 ;{ }^{*} p<0,001 ;{ }^{\&} p=0,012$. MEEM: Mini-Exame do Estado Mental; TDR: Teste do Desenho do Relógio (escore de Schulman); EDB: Escala de Demência de Blessed. 1: em anos; 2: saláriosmínimos. 
$\mathrm{Na}$ amostra total, sujeitos com evidência de declínio cognitivo e história prévia tiveram maior frequência de autopercepção negativa do estado de saúde $\left(\chi^{2}=7,4, \mathrm{df}=1, \mathrm{p}=0.006 ; \chi^{2}=4,9, \mathrm{df}=1\right.$, $p=0,027$, respectivamente). Os indivíduos com autopercepção negativa da saúde tiveram maiores escores na Escala de Demência de Blessed ( $p=0,021)$, indicando maior comprometimento cognitivo nessa população.

Nos pacientes com demência, aqueles com autopercepção de saúde positiva tiveram maior pontuação no IQCODE, indicando maior gravidade do quadro demencial de acordo com a observação de seus familiares/cuidadores. Neste grupo de sujeitos, a autopercepção de saúde não esteve significativamente relacionada às outras variáveis avaliadas (Tabelas 2 e 3 ). Já nos sujeitos sem evidência de quadro demencial, história prévia de eventos cerebrovasculares se relacionou à pior autopercepção da saúde $\left(\mathrm{OR}=1,15 \mathrm{CI}_{95 \%}[1,01-\right.$ $1,32], \mathrm{p}=0,027)$.

\section{Discussão}

No presente estudo, o diagnóstico de demência se associou à menor atividade física, à menor participação em atividades de lazer e à maior frequência de autopercepção negativa de seu estado de saúde. Nos pacientes com declínio cognitivo/demência, a autopercepção positiva do estado de saúde se associou a maiores escores no IQCODE. No conhecimento dos autores, não há trabalhos anteriores no Brasil que avaliaram a autopercepção da saúde e a sua relação com desempenho cognitivo e diagnóstico de declínio cognitivo/demência em idosos. A elevada prevalência de casos de declínio cognitivo/demência nesta população $(26 \%)$ é muito superior à observada em estudos epidemiológicos no Brasil ${ }^{26}$. Esse fato pode ser devido a viés de amostragem (amostra de conveniência no presente estudo), assim como viés de testagem, já que os testes cognitivos usados neste estudo sofrem influência significativa da escolaridade ${ }^{27-29} \mathrm{e}$, portanto, devem ser analisados com cuidado.

A autopercepção da saúde implica julgamento do sujeito sobre seu estado de saúde, processo que sofre influência da capacidade cognitiva do sujeito. No idoso, a autopercepção da saúde se constrói dentro de um contexto que envolve as alterações físicas e mentais próprias ao processo de envelhecimento, associadas a questões culturais e ambientais no qual o idoso está inserido ${ }^{1,2}$. Na amostra total, a autopercepção negativa de saúde se associou ao diagnóstico de provável declínio cognitivo/demência e à história de eventos cerebrovasculares. Entretanto, a análise para cada grupo diagnóstico revelou um padrão distinto de resultados. Nos idosos com provável declínio cognitivo/demência, aqueles com autopercepção positiva da sua saúde tiveram maiores escores no IQCODE, indicando maior gravidade do quadro demencial. Esse achado sugere que, em idosos com demência, a medida que o quadro demencial avança ocorre uma diminuição da capacidade de insight e de integrar de modo adequado tanto estímulos do ambiente quanto estímulos internos ${ }^{30}$. Portanto, ele avaliaria de modo positivo seu estado de saúde apesar das limitações decorrentes de problemas físicos e do próprio quadro demencial. Entre os idosos sem demência, aqueles com eventos cerebrovasculares prévios tiveram mais frequentemente autopercepção negativa da saúde. Tal achado está em linha com estudos anteriores em que sujeitos portadores de doenças crônicas (incluindo as doenças cerebrovasculares) têm mais comumente uma autopercepção negativa do estado de saúde ${ }^{3}$.

No conhecimento dos autores, este é o primeiro estudo no Brasil que demonstrou uma associação positiva entre quadros demenciais e autopercepção negativa de saúde. Além disso, o diagnóstico de declínio cognitivo/demência se associou a menor nível de atividade física e de participação em atividades recreativas. Esses resultados estão em concordância com diversos estudos na literatura nos quais idosos com estilo de vida sedentária e menor participação em atividades comunitárias/sociais apresentaram maior risco de desenvolver quadros demenciais $\mathrm{s}^{31-32}$. Entretanto, o desenho transversal desse estudo, a amostra não randomizada, a utilização apenas de testes de triagem de demência, assim como o pequeno número de sujeitos limitam a interpretação desses resultados e outros estudos são necessários para a confirmação entre a associação de autopercepção negativa de saúde e o diagnóstico de demência.

Em conclusão, este é o primeiro estudo brasileiro que relaciona autopercepção negativa da saúde à cognição em idosos. Mostrou-se que a autopercepção negativa da saúde está relacionada a maior risco de declínio cognitivo em idosos. Além disso, entre os pacientes com declínio cognitivo, a autopercepção positiva de saúde está associada à maior gravidade dos déficits cognitivos, refletindo, possivelmente, uma menor capacidade de crítica quanto ao estado mórbido.

\section{Agradecimentos}

À equipe de saúde do posto Amílcar Viana Martins e à comunidade abrangida pelo posto.

BSD é bolsista de doutorado da Coordenação de Aperfeiçoamento de Pessoal de Nível Superior (Capes), Brasil. DHMF, FCAC, LQL, CRS e CBF são bolsistas de iniciação científica do Conselho Nacional de Desenvolvimento Científico e Tecnológico (CNPq), da Fundação de Amparo à Pesquisa do Estado de Minas Gerais (Fapemig) e PROEX (Pró-Reitoria de Extensão).

Tabela 2. Diagnóstico de declínio/cognitivo, variáveis sociodemográficas e escores nos testes cognitivos de acordo com a autopercepção de saúde

\begin{tabular}{l|c|c|c|c|c|c|c|c|c}
\hline $\begin{array}{c}\text { Autopercepção } \\
\text { de saúde }\end{array}$ & $\begin{array}{c}\text { Declínio cognitivo } \\
\text { (sim/não) }\end{array}$ & Idade (anos) & $\begin{array}{c}\text { Escolaridade } \\
\text { (anos) }\end{array}$ & $\begin{array}{c}\text { Renda } \\
\text { familiar }{ }^{1}\end{array}$ & $\begin{array}{c}\text { Número de } \\
\text { medicações }\end{array}$ & MEEM & TDR & IQCODE & EDB \\
\hline Positiva & $7 / 42$ & $76,2 \pm 6,1$ & $3,2 \pm 2,2$ & $2,7 \pm 1,7$ & $3,4 \pm 2,4$ & $21,6 \pm 5,3$ & $1,8 \pm 1,3$ & $3,3 \pm 0,4$ & $1,0 \pm 2,4$ \\
\hline Negativa & $19 / 30^{*}$ & $77,5 \pm 5,8$ & $3,6 \pm 2,5$ & $2,5 \pm 1,7$ & $4,4 \pm 2,8$ & $20,5 \pm 4,9$ & $1,4 \pm 1,5$ & $3,4 \pm 0,4$ & $2,2 \pm 2,8^{* *}$ \\
\hline
\end{tabular}

${ }^{*} p=0,006 ;{ }^{* *} p=0,21$. MEEM: Mini-Exame do Estado Mental; TDR: Teste do Desenho do Relógio (escore de Schulman); EDB: Escala de Demência de Blessed. 1: salários-mínimos.

Tabela 3. Variáveis sociodemográficas e escores nos testes cognitivos nos pacientes com demência e sem demência de acordo com a autopercepção de saúde

\begin{tabular}{|c|c|c|c|c|c|c|c|c|}
\hline $\begin{array}{l}\text { Autopercepção } \\
\text { de saúde }\end{array}$ & Idade' $^{1}$ & Escolaridade $^{1}$ & Renda familiar ${ }^{2}$ & $\begin{array}{l}\text { Número de } \\
\text { medicações }\end{array}$ & MEEM & TDR & IOCODE & EDB \\
\hline \multicolumn{9}{|c|}{ Demência } \\
\hline Positiva & $84,3 \pm 9,9$ & $3,1 \pm 1,7$ & $2,3 \pm 1,1$ & $3,0 \pm 1,3$ & $14,0 \pm 7,1$ & $0,7 \pm 0,7$ & $4,2 \pm 0,5^{*}$ & $3,9 \pm 5,5$ \\
\hline Negativa & $80,0 \pm 6,1$ & $3,9 \pm 2,8$ & $1,9 \pm 1,0$ & $4,1 \pm 2,2$ & $17,2 \pm 4,5$ & $0,8 \pm 0,8$ & $3,5 \pm 0,6$ & $4,3 \pm 3,5$ \\
\hline \multicolumn{9}{|c|}{ Sem demência } \\
\hline Positiva & $74,9 \pm 4,1$ & $3,2 \pm 2,3$ & $2,8 \pm 1,7$ & $3,5 \pm 2,5$ & $22,8 \pm 3,8$ & $2,0 \pm 1,3$ & $3,1 \pm 0,2$ & $0,5 \pm 0,8$ \\
\hline Negativa & $75,9 \pm 5,2$ & $3,5 \pm 2,4$ & $2,8 \pm 2,0$ & $4,6 \pm 3,1$ & $22,6 \pm 3,4$ & $1,9 \pm 1,7$ & $3,2 \pm 0,2$ & $0,9 \pm 1,0$ \\
\hline
\end{tabular}

\footnotetext{
${ }^{*} p$ = 0,026; MEEM: Mini-Exame do Estado Mental; TDR: Teste do Desenho do Relógio (escore de Schulman); EDB: Escala de Demência de Blessed. 1: em anos; 2: salários-mínimos.
} 


\section{Referências}

1. Lima-Costa MF, Firmo JOA, Uchoa E. A estrutura da auto-avaliação da saúde entre idosos: projeto Bambuí. Rev Saúde Pública. 2004;38(6):827-34.

2. Lima-Costa MF, Peixoto SV, Matos DL, Firmo JOA, Uchoa E. A influên cia de respondente substituto na percepção da saúde de idosos: um estudo baseado na Pesquisa Nacional por Amostra de Domicílios (1998, 2003) e na coorte de Bambuí, Minas Gerais, Brasil. Cad Saúde Pública. 2007;23(8):1893-902.

3. Alves LS, Rodrigues RN. Determinantes da autopercepção de saúde entre idosos do Município de São Paulo, Brasil. Rev Panam Salud Publica. 2005; 17(5/6):333-41

4. Ofstedal MB, Zimmer Z, Cruz G, Chan A, Lin YH. Self-assessed health expectancy among older Asians: a comparison of Sullivan and multistate life table methods. Ann Arbor: University of Michigan, Population Studies Center; 2002

5. Kaplan GA, Camacho T. Perceived health and mortality: a nine-year follow-up of the human population laboratory cohort. Am J Epidemiol. 1983;117(3):292-304

6. Idler EL, Benyamini Y. Self-rated health and mortality: a review of twentyseven community studies. J Health Soc Behav. 1997;38(1):21-37.

7. Marcellini F. Health perception of elderly people: the results of a longitudinal study. Arch Gerontol Geriatr Suppl. 2002;35(Suppl):181-9.

8. Appels A, Bosma H, Grabauskas V, Gostautas A, Sturmans F. Self-rated health and mortality in a Lithuanian and a Dutch population. Soc Sci Med. 1996;42(5):681-9.

9. Alves LS, Rodrigues RN. Determinantes da autopercepção de saúde entre idosos do Município de São Paulo, Brasil [dissertação]. Belo Horizonte (MG): Universidade Federal de Minas Gerais; 2004.

10. Instituto Brasileiro de Geografia e Estatística. Disponível em: http://www. ibge.gov.br. Acesso em: 1 de outubro de 2008.

11. Kalache A, Veras RP, Ramos LR. Envelhecimento da população mundial: um desafio novo. Rev Saúde Pública. 1987;21:200-10.

12. Lopes MA, Hototian SR, Reis GC, Elkis H, Bottino CMC. Systematic review of dementia prevalence 1994 to 2000. Dement Neuropsychol. 2007;3:230-40.

13. Nitrini R, Caramelli P, Herrera Jr. E, et al. Incidence of dementia in a community-dwelling Brazilian population. Alzheimer Dis Assoc Disord. 2004; $18: 241-6$

14. Garrido R, Almeida OP. Distúrbios de comportamento em pacientes com demência: impacto sobre a vida do cuidador. Arq Neuro-Psiquiatr. 1999;57(2B):427-34.

15. Dachs J, Norberto W. Determinantes das desigualdades na auto-avaliação do estado de saúde no Brasil: análise dos dados da PNAD/1998. Ciên Saúde Colet. 2002;7(4)641-57.

16. Diniz BSO, Volpe FM, Tavares AR. Nível educacional e idade no desempenho no Mini-Exame do Estado Mental em idosos residentes na comunidade. Rev Psiq Clín. 2007;34:13-7.
17. Questionnaire boas: Brazil Old Age Schedule (Multidimensional questionnaire for the elderly population). Rio de Janeiro, Instituto de Medicina Social da UERJ; 1987.

18. Veras RP. País jovem com cabelos brancos: a saúde do idoso no Brasil. Rio de Janeiro: Relume Dumará; 1994.

19. Veras RP, Souza CAM, Cardoso RF, Milioli R, Silva SD. Pesquisando populações idosas - A importância do instrumento e o treinamento de equipe: uma contribuição metodológica. Rev Saúde Pública. 1988;22(6):513-8.

20. Jorm AF, Scott R. Performance of the Informant Questionnaire on Cognitive Decline in the Elderly (IQCODE) as a screening test for dementia. Psychol Med. 1991;21:785-90.

21. Abreu ID, Nunes PV, Diniz BS, Forlenza OV. Combining functional scales and cognitive tests in the screening for mild cognitive impairment at a university-based memory clinic in Brazil. Rev Bras Psiquiatr. 2008;30(4):346-9.

22. Shulman KI, Shedletsky R, Silver IL. The chalenge of time: clockdrawing and cognitive function in the elderly. Int J Geriatr Psychiatry. 1986;1(2):135-40. doi: 10.1002/gps.930010209

23. Blessed G, Tomlison BE, Roth $\mathrm{M}$. The association between quantitative measures of dementia and of senile change in the cerebral grey matter of the elderly subjects. Psychiatry. 1968;114:797-811.

24. Folstein MF, Folstein SE, McHugh PR. "Mini-Mental State": a practical method for grading the cognitive state of patients for the clinician. J Psychiatr Res. 1975;12:189-98.

25. Almeida OP. Mini-Exame do Estado Mental e o diagnóstico de demência no Brasil. Arq. Neuropsiquiatr. 1998;56(3B):605-12.

26. Herrera E, Caramelli P, Silveira ASB, Nitrini R. Epidemiologic survey of dementia in a community-dwelling Brazilian population. Alzheim Dis Ass Disord. 2002;16:103-8.

27. Farmer ME, Kittner SJ, Rae DS, Bartko JJ, Regier DA. Education and change in cognitive function: the Epidemiologic Catchment Area study. Ann Epidemiol. 1995; 5:1-7.

28. Jorm AF, Scott R, Henderson AS, Kay DW. Educational level differences on the Mini-Mental State: the role of test bias. Psychol Med. 1988;18: 727-31.

29. Snowdon DA, Ostwald SK, Kane RL. Education, survival, and independence in elderly Catholic sisters, 1936-1988. Am J Epidemiology. 1989;130:999-1012.

30. Hodges JR. Alzheimer's centennial legacy: origins, landmarks and the current status of knowledge concerning cognitive aspects. Brain. 2006;129:2811-22. doi:10.1093/brain/awl275

31. Verghese J, Lipton RB, Katz MJ, et al. Leisure activities and the risk of dementia in the elderly. N Engl J Med. 2003;348:2508-16.

32. Wilson RS, Bennett DA, Bienias JL, et al. Cognitive activity and incident $\mathrm{AD}$ in a population-based sample of older persons. Neurology. 2002;59:1910-4. 